regions also thought to be late in replicating their DNA. Kato also recorded the sites where spontaneous breakage occurred and it turned out that these sites are the same as those broken by the virus.

But what do these results indicate about the nature of the chromosome at the points of breakage or about the properties of the virus? It seems likely that there is some qualitative difference in the structure of the chromosome that predisposes certain regions to high breakage frequencies. Some years ago Hsu proposed that regions broken by certain mutagens were rich in particular DNA bases, but since then knowledge of this problem has not been much extended. The position in the infected cell from which the virus directs the induction of a chromosome break is not even known with certainty. Observations by Hälkka (Hereditas, 58, 248 and 251 ; 1967) may be relevanthe found that in the testes of the cricket Acheta infected by a rickettsia-like organism, the microbe was to be observed lying on the nuclear envelope and even to be lying on the chromosomes themselves. Infection of these meiotic cells caused many lesions in the chromosomes similar to those induced by viruses. If a parallel can be drawn, it is that the damage done by viruses to chromosomes may well be caused by their intimate association with the chromosome at certain preferred points, thereby impairing chromosome replication. Unfortunately, viruses have not yet been located within the structure of chromosomes from higher organisms.

\section{Steroids and ATPase}

\section{from our Medical Biochemistry Correspondent}

THE maintenance of the correct concentrations of sodium and potassium ions in mammalian systems is literally of vital importance. When sodium and potassium ions are moved across cell membranes against a concentration gradient, adenosine triphosphate (ATP) is split to provide the energy for the process. The enzyme responsible is thought to be an A'TPase which is dependent on the presence of sodium and potassium ions for its activity. Bader, Post and Bond have now made preparations of this enzyme from eleven different species and six different tissues, and have shown that they are remarkably similar (Biochim. Biophys. Acta, 150,$41 ; 1968)$. They all required the presence of sodium ions to form a ${ }^{32} \mathrm{P}$-labelled protein from ${ }^{32} \mathrm{P}$-labelled ATP, and dephosphorylation of the protein was always stimulated by the presence of potassium ions. Although the specifie activity of the preparations varied greatly, the ratio of ATPase activity to phosphoenzyme and ATPase to acyl phosphatase was always approximatcly the same. The ${ }^{32}$ P-labelled preparations were digested with pepsin or pronase and the resulting ${ }^{32}$ P.labelled peptides were separated by electrophoresis. The peptides all behaved identically on electrophoresis and on treatment with hydroxylamine, suggesting that the enzyme structure which becomes phosphorylated is the same in preparations of the enzyme from all these different sources.

The corticosteroids produced by the adrenal gland are the hormones which regulate salt and water meta. bolism, and it has now been shown that the activity of the sodium and potassium activated ATPase in rat kidney varies with the plasma concentration of adrenal steroids (P. I. Jorgensen, Biochim. Biophys.
Acta, 151, 212; 1968). After removal of the adrenals, the activity of the ouabain-sensitive sodium and potassium activated ATPase activity of rat kidney decreased along with the sodium/potassium ratio of the plasma. A sodium-rich diet delayed the appearance of both decreases. The decrease in activity appeared to be due to a decrease in the amount of enzyme, as the enzyme kinetics were the same for preparations from normal and adrenalectomized animals. The attachment of the enzyme to the membranes of the microsomes may be altered, as the decrease in activity was most marked when activity was measured in the presence of deoxycholate and ethylene diamine tetra-acetic acid.

If adrenal steroids affect this ATPase, and these enzymes are all very similar, it is hardly surprising that adrenal steroids can affect the salt metabolism of tissues other than the kidney. In a recent investigation (R. Shields, J. B. Miles and C. Gilbertson, Brit. Med.J., (i), 93; 1968) a patient with an adrenal tumour secreted four to five times as much potassium into the colon as did eight normal controls, but 3 months after operation his potassium loss was normal. Since half the daily loss of potassium from this patient was in the faeces, urinary estimations alone could seriously underestimate the potassium loss in patients treated with adrenal steroids, with serious consequences.

\section{Ribosomal Proteins}

\section{from our Cell Biology Correspondent}

THe chemical heterogeneity of ribosomal protein has now been established beyond question. Tissières and his colleagues, for example (Proc. US Nat. Acad. Sci., $5 \%, 1294 ; 1967)$, recently isolated and partially purified a number of proteins from $E$. coli ribosomes. Given the great chemical heterogeneity of ribosomal proteins, there is every reason to expect that these proteins are functionally heterogeneous. The problem then is that of defining the functions of the individual proteins and how their individual properties relate to the overall function of the ribosome. In the current Proc. US Nat. Acad. Sci. $(58,2430 ; 1967)$, Traub, Hosokawa, Craven and Nomura report an analysis of the function of five basic proteins isolated from the $30 S$ sub-unit of $E$. coli ribosomes.

Their approach was to prepare a series of ribosome derivatives, each deficient in a specific ribosomal protein and to compare the properties of their derivatives with those of normal ribosomes. Having first dissociated $70 \mathrm{~S}$ ribosomes into $30 \mathrm{~S}$ and $50 \mathrm{~S}$ sub-units, Traub et al. further dissociated the $30 \mathrm{~S}$ sub-unit into a $23 \mathrm{~S}$ core which has none of the properties of a $30 S$ sub-unit, and free protein-the so-called split proteins. They then separated the split proteins into acidic and basic protein fractions. Particles reconstituted from $23 S$ cores and the acidic split proteins have none of the known properties of $30 S$ sub-units, whereas particles reconstituted from cores and the basic split proteins are partially active. The basic split proteins are essential for the $30 S$ functions, whereas the acidic split proteins, though stimulatory, are not essential.

Following up this result, Traub et al. resolved the basic split protein into five component proteins with quite distinct amino-acid compositions. To determine the function of each of these five proteins, they reconsti- 